\title{
BreastCare
}

\section{Vol 1, Issue 2, April 2006}

Focus: Trastuzumab

Editorials

64 Trastuzumab as Intellectual, Medical and Economical Challenge

Zielinski, C.C. (Wien)

66 Trastuzumab for Early Breast Cancer:

Hope or Hype?

Smith, I.E. (London)

Review Articles

69 HER2 Testing in Breast Cancer: Opportunities and Challenges

Lebeau, A. (München)

78 Trastuzumab (Herceptin ${ }^{\circledR}$ ): Monoclonal Antibody in the Treatment of HER2/neu-Overexpressing Breast Cancer in the Metastatic and (Neo)adjuvant Situation

Ditsch, N.; Rückert, S.; Kümper, C.; Lenhard, M.; Kahlert, S.; Bauerfeind, I.; Friese, K. (München); Untch, M. (Berlin)

86 Current Status of Financing and Reimbursement of Trastuzumab (Herceptin ${ }^{\circledR}$ ) for Adjuvant and Advanced Therapy of Breast Cancer in Germany Jacobs, V. (München)

Expert Discussion

96 Adjuvant Treatment with Trastuzumab in Clinical Practice Outside Clinical Studies Chair: Greil, R. (Salzburg)

Focus: SABCS 2005

Review Articles

103 Familial Breast Cancer, Prevention, Risk Factors and Genetics - Highlights from the San Antonio Breast Cancer Symposium (SABCS) Poster Presentations

Bauerschlag, D.O.; Schem, C.; Mundhenke, C.; Meinhold-Heerlein, I.; Jonat, W.; Maass, N. (Kiel)
Band 1, Heft 2, April 2006

\section{Schwerpunkt: Trastuzumab}

Editorials

64 Trastuzumab als intellektuelle, medizinische und ökonomische Herausforderung

Zielinski, C.C. (Wien)

66 Trastuzumab beim frühen Mammakarzinom: Hoffnung oder heiße Luft? Smith, I.E. (London)

Übersichtsarbeiten

69 HER2-Bestimmung beim Mammakarzinom: Möglichkeiten und Herausforderungen Lebeau, A. (München)

78 Trastuzumab (Herceptin ${ }^{\circledR}$ ): Antikörpertherapie beim HER2/neu-überexprimierenden Mammakarzinom in der metastasierten und (neo)adjuvanten Situation

Ditsch, N.; Rückert, S.; Kümper, C.; Lenhard, M.; Kahlert, S.; Bauerfeind, I.; Friese, K. (München); Untch, M. (Berlin)

86 Aktueller Stand von Finanzierung und Kostenerstattung von Trastuzumab (Herceptin ${ }^{\circledR}$ ) für die adjuvante und fortgeschrittene Therapie des Mammakarzinoms in Deutschland Jacobs, V. (München)

Expertenplenum

96 Adjuvanter Einsatz von Trastuzumab in der klinischen Praxis ausserhalb von klinischen Studien Gesprächsleitung: Greil, R. (Salzburg)

\section{Schwerpunkt: SABCS 2005}

Übersichtsarbeiten

103 Familiärer Brustkrebs, Prävention, Risikofaktoren und genetische Grundlagen - Höhepunkte der Posterpräsentationen vom San Antonio Breast Cancer Symposium (SABCS)

Bauerschlag, D.O.; Schem, C.; Mundhenke, C.; Meinhold-Heerlein, I.; Jonat, W.; Maass, N. (Kiel) 
108 San Antonio Report 2005: Diagnostics, Detection, Proteomics, Nanotechnology, Pathology, Operative Techniques

Paepke, S.; Euler, U.; Baumgärtner, A.K.; Schwarz-Boeger, U.; Harbeck, N.; Jacobs, V.R. (München)

113 Tumor Cell Dissemination and Metastasis in Breast Cancer: Selected Presentations at the 28th San Antonio Breast Cancer Symposium 2005 Müller, V. (Hamburg); Stickeler, E. (Freiburg i.Br.)

118 Predicitve and Prognostic Markers in Breast Cancer Treatment - Presentations at the 28th San Antonio Breast Cancer Symposium 2005 Rody, A.; Karn, T.; Kaufmann, M. (Frankfurt/M.)

124 The Endocrine Origin and Different Characters of Breast Cancers - Recent Research on Hormone Receptors and Endocrine Treatment Dubsky, P.; Gnant, M. (Wien)

129 Advances in Adjuvant and Primary Systemic Therapy of Early Breast Cancer Bischoff, J.; Costa, S.D. (Magdeburg)

Informations from Breast Centers

134 The Southwest Breast Cancer Center (SWBC) Heidelberg - a Network to Improve Quality of Breast Cancer Diagnosis and Treatment Schneeweiss, A.; Wacker, J.; Sohn, C. (Heidelberg)

Breast Cancer Organisation

136 Brustkrebs Deutschland e.V.

Haidinger, R. (München)

The Breast in Art and Literature

138 The Curved Stroke of the Brush Reveals the True Master

Müller, H.J. (Freiburg i.Br.)

140 PharmaNews / PharmaTicker

141 Meetings and Conferences

137 Imprint

143 Guidelines for Authors

Forthcoming Papers are listed on page 144.
108 Bericht vom SABCS 2005: Mammakarzinom Diagnostik, Erkennung, Proteomics, Pathologie, operative Techniken

Paepke, S.; Euler, U.; Baumgärtner, A.K.; Schwarz-Boeger, U.; Harbeck, N.; Jacobs, V.R. (München)

113 Tumorzelldisseminierung und Metastasierung beim Mammakarzinom: Ausgewählte Ergebnisse vom 28. San Antonio Breast Cancer Symposium 2005 Müller, V. (Hamburg); Stickeler, E. (Freiburg i.Br.)

118 Prädiktive und prognostische Marker beim Mammakarzinom - Präsentationen auf dem 28. San Antonio Breast Cancer Symposium 2005 Rody, A.; Karn, T.; Kaufmann, M. (Frankfurt/M.)

124 Die endokrine Herkunft und die unterschiedlichen Typen von Brustkrebs - aktuelle Forschungsergebnisse über Hormonrezeptoren und endokrine Therapieansätze Dubsky, P.; Gnant, M. (Wien)

129 Fortschritte in der adjuvanten und primär systemischen Therapie des Mammakarzinoms Bischoff, J.; Costa, S.D. (Magdeburg)

Informationen aus den Brustzentren

134 Das Südwestdeutsche Brustcentrum (SWBC) Heidelberg - ein Netzwerk zur Qualitätssteigerung in der Diagnostik und Therapie des Mammakarzinoms Schneeweiss, A.; Wacker, J.; Sohn, C. (Heidelberg)

Brustkrebsorganisation

136 Brustkrebs Deutschland e.V. Haidinger, R. (München)

Die Brust in Kunst und Literatur

138 An dem, was rund ist, zeigt sich der Meister Müller, H.J. (Freiburg i.Br.)

140 PharmaNews / PharmaTicker

141 Tagungen und Kongresse

137 Impressum

143 Hinweise für Autoren

Einen Ausblick auf den Inhalt der kommenden Hefte finden Sie auf Seite 144. 